\title{
Comunicación
}

\section{Detección de Anticuerpos Contra Toxoplasma gondii en un Hurón Doméstico (Mustela putorius furo) en Lima, Perú}

\author{
Detection of Antibodies Against Toxoplasma gondii in a Domestic Ferret \\ (Mustela putorius furo) in Lima, PerU
}

Jesús Lescano G. ${ }^{1,2}$, Miryam Quevedo U. ${ }^{1}$

\section{Resumen}

Se reconoce que los hurones domésticos (Mustela putorios furo) son susceptibles a la toxoplasmosis, pero la información al respecto es escasa. Se describe el caso de un hurón hembra de 6.5 años de edad llevado a consulta a la Clínica de Animales Silvestres y Exóticos de la Universidad Nacional Mayor de San Marcos (Lima, Perú), por presentar anorexia y diarrea reciente. El examen físico reveló deshidratación, bradicardia, hipotermia y letargia. El examen hematológico reveló leucocitosis, neutrofilia y trombocitopenia. El examen bioquímico sérico reveló hipoglucemia y elevación de la creatina quinasa. Se detectó IgG e IgM anti-Toxoplasma mediante la prueba de Hemaglutinación Indirecta. El hurón falleció dos días después de iniciar la terapia y no se permitió realizar el examen post mortem.

Palabras clave: Mustelidae, toxoplasmosis, inmunoglobulinas, protozoario, serología

\section{Abstract}

It is known that domestic ferrets (Mustela putorios furo) are susceptible to toxoplasmosis; however, information is scarce. This report describes the case of a 6.5 years old female ferret received at the Wild and Exotic Animals Clinic of the Universidad Nacional Mayor de San Marcos (Lima, Peru), which presented recent anorexia and diarrhoea. Physical examination revealed dehydration, bradycardia, hypothermia, and lethargy. Haematology revealed leucocytosis, neutrophilia, and thrombocytopenia. Serum biochemistry revealed hypoglycaemia and increased creatine kinase. Anti-Toxoplasma

\footnotetext{
${ }^{1}$ Laboratorio de Anatomía Animal y Fauna Silvestre, Facultad de Medicina Veterinaria, Universidad Nacional Mayor de San Marcos, Lima, Perú

${ }^{2}$ E-mail: xtianlescano@gmail.com
}

Recibido: 26 de julio de 2016

Aceptado para publicación: 14 de noviembre de 2016 
IgM and IgG were found by Indirect Hemagglutination assay. The ferret died two days after therapy was established and post mortem examination was not allowed.

Key words: Mustelidae, toxoplasmosis, immunoglobulins, protozoa, serology

\section{INTRODUCCIÓN}

Virtualmente, todos los animales vertebrados homeotérmicos pueden ser hospederos intermediarios de Toxoplasma gondii; sin embargo, la evidencia indica que los carnívoros presentan mayor predisposición a la infección, lo cual se refleja en la detección de altas seroprevalencias en este grupo taxonómico (Smielewska-Los et al., 2000; Dubey y Odening, 2001; Miller, 2008). Los miembros de la familia Felidae (incluyendo al gato doméstico) son hospederos definitivos y rara vez desarrollan la enfermedad clínica (Tenter et al., 2000), habiéndose reportado únicamente casos correspondientes a individuos mantenidos en cautiverio (Dubey y Odening, 2001).

Se han detectado anticuerpos contra $T$. gondii en felinos silvestres de vida libre como jaguares (Panthera onca), linces ibéricos (Lynx pardinus), linces rojos (Lynx rufus) y gatos silvestres (Felis silvestris) (Sobrino et al., 2007; García-Bocanegra et al., 2010; Onuma et al., 2014). Entre otros carnívoros, se han observado casos de toxoplasmosis clínica en prociónidos (e.g. Procyon lotor), cánidos (e.g. Canis latrans, Vulpes vulpes, Urocyon cinereoargenteus), mefítidos (Mephitis mephitis) y úrsidos (e.g. Ursus maritimus, Ursus arctos) (Dubey y Odening, 2001; Collins, 2015).

En mustélidos (Orden Carnivora, Familia Mustelidae) silvestres, se ha detectado evidencia serológica de infección por $T$. gondii en Neovison vison, Lontra canadensis, Martes americana y Martes pennanti en países del hemisferio norte (e.g. Estados Unidos, Canadá e Irlanda) (Tizard et al., 1976; O'Crowley y Wilson, 1991; Tocidlowski et al., 1997). La infección por toxoplasmosis en hurones domésticos (Mustela putorius furo) ha sido registrada desde 1932 (Coutelen, 1932, citado por Burns et al., 2003); sin embargo, los reportes de casos son escasos (Morrisey, 1996; Burns et al., 2003; Lewington, 2007; Mentré, 2012).

T. gondii ha sido detectado mediante histopatología en hurones de laboratorio (Lewington, 2007). Asimismo, la infección congénita en hurones ha sido sugerida con base en los casos de muerte súbita de hurones neonatos (incluyendo animales de 1 día de edad) en criaderos de Estados Unidos y Nueva Zelanda, en los cuales se observaron áreas de necrosis en pulmones, corazón e hígado con presencia de organismos compatibles con Toxoplasma (Thornton y Cook, 1986; Lewington, 2007). El presente artículo tuvo por objetivo reportar la detección de anticuerpos contra Toxoplasma gondii en un hurón doméstico por primera vez en Perú y describir su presentación clínica.

\section{Caso Clínico}

Se recibió un hurón (Mustela putorius furo) hembra, esterilizada, de 6.5 años de edad, en la Clínica de Animales Silvestres y Exóticos de la Facultad de Medicina Veterinaria, Universidad Nacional Mayor de San Marcos, Lima, Perú.

El animal fue llevado a consulta debido a que se mostraba decaído, anoréxico y presentaba heces pastosas. Al examen físico, el animal pesaba $825 \mathrm{~g}$ y presentaba buena condición corporal (3/5). Además, presentaba 
Cuadro 1. Resultados de los exámenes hematológicos y bioquímico-séricos realizados al hurón (Mustela putorius furo) llevado a consulta con un cuadro de anorexia y decaimiento

\begin{tabular}{|c|c|c|}
\hline Analito $^{1}$ & Resultado & Rango referencial $^{2}$ \\
\hline Eritrocitos $\left(10^{6} / \mu \mathrm{l}\right)$ & 8.7 & $6.77-9.76$ \\
\hline Hemoglobina (g/dl) & 17.8 & $15.2-17.4$ \\
\hline Hematocrito (\%) & 51 & $42-55$ \\
\hline $\operatorname{VCM}(\mathrm{fL})$ & 58.6 & $50-54$ \\
\hline $\operatorname{HCM}(\mathrm{g} / \mathrm{dl})$ & 20.5 & $15-18$ \\
\hline CHCM (g/dl) & 34.9 & $32-35$ \\
\hline Plaquetas $\left(10^{3} / \mu \mathrm{l}\right)$ & 271 & $310-910$ \\
\hline Leucocitos $\left(10^{3} / \mu 1\right)$ & 13.1 & $2.5-8.6$ \\
\hline Segmentados (células/ $\mu 1)$ & 10,218 & $725-2409$ \\
\hline Linfocitos (células/ $\mu \mathrm{l}$ ) & 2,882 & $1475-5590$ \\
\hline Mono citos (células/ $\mu \mathrm{l}$ ) & 0 & $100-372$ \\
\hline 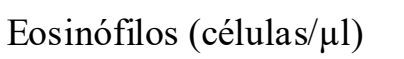 & 0 & $50-516$ \\
\hline Basófilos (células/ $\mu 1$ ) & 0 & $0-172$ \\
\hline Creatinina (mg/dl) & 0.6 & $0.2-0.6$ \\
\hline $\operatorname{ALT}(\mathrm{UI} / \mathrm{L})$ & 69 & $82-289$ \\
\hline Glucosa (mg/dl) & 44 & $62-134$ \\
\hline Creatina quinasa (UI/l) & 399 & $55-93$ \\
\hline
\end{tabular}

${ }^{1}$ VCM: volumen corpuscular medio; HCM: contenido corpuscular medio de hemoglobina; $\mathrm{CHCM}$ : concentración de hemoglobina corpuscular media; ALT: alanina aminotransferasa

${ }^{2}$ Rangos referenciales tomados de Quesenberry y Orcutt (2012)

deshidratación (8\%), bradicardia (601pm), hipotermia $\left(35.9^{\circ} \mathrm{C}\right)$, frecuencia respiratoria de 36 rpm y letargia. Se solicitó realizar examen ecográfico abdominal, radiografía de tórax, hematología y bioquímica sérica.

La ecografía reveló ascitis leve, esplenomegalia, nefropatía bilateral y sospecha de congestión venosa hepática. La radiografía reveló disminución de la imagen hepática. Se administró terapia de soporte con lactato de Ringer (200 ml/kg SC), suplemento nutricional (Hemolitan, Vetnil, Brasil), dieta blanda (Hills a/d Prescription Diet, Hils Pet Nutrition, EEUU) y enrofloxacina $(30 \mathrm{mg} / \mathrm{kg}$ SC q24h) (Baytril, Bayer, Alemania)
(Carpenter y Marion, 2012). El examen hematológico reveló trombocitopenia, leucocitosis y neutrofilia.

El examen bioquímico sérico reveló hipoglucemia e incremento de la creatina quinasa. Los resultados de los exámenes hematológicos y bioquímico séricos se detallan en el Cuadro 1. Según estos resultados, se solicitó descartar toxoplasmosis.

Al día siguiente, el animal seguía presentando bradicardia e hipotermia. Además, presentó disnea y seguía defecando pastoso. Se administró tratamiento similar al día anterior y se añadió amoxicilina $(25 \mathrm{mg} / \mathrm{kg} \mathrm{SC}$ 
q12h) (Carpenter y Marion, 2012). La prueba de Hemaglutinación Indirecta y titulación con mercaptoetanol (Toxotest, Wiener Lab, Argentina) reveló la presencia de anticuerpos contra $T$. gondii, con titulaciones de 1/2048 ( $\sin$ mercaptoetanol) y $1 / 512$ (con mercaptoetanol). De acuerdo a este resultado, se modificó la terapia antibiótica por clindamicina $(12.5 \mathrm{mg} / \mathrm{kg}$ SC q12h) (Carpenter y Marion, 2012). Dos días más tarde, el paciente falleció y el propietario no autorizó realizar el examen post mortem.

\section{Discusión}

De acuerdo al conocimiento de los autores, el presente artículo reporta por primera vez la detección de anticuerpos contra $T$. gondii en un hurón en Perú. Entre las limitaciones del presente reporte, se puede destacar la imposibilidad de realizar el examen post mortem completo a fin de obtener el diagnóstico definitivo de la infección por $T$. gondii mediante técnicas auxiliares, como histopatología, inmunohistoquímica y PCR (Burns et al., 2003; Mentré, 2012). Asimismo, la prueba diagnóstica empleada en el presente caso no ha sido específicamente validada en hurones criados en cautiverio, aunque ha sido previamente empleada en primates no humanos (Familias Cebidae, Aotidae, Atelidae, Pitheciidae, Homonidae y Cercophiteciidae), carnívoros (Familias Felidae, Ursidae, Mustelidae y Procyonidae) $\mathrm{y}$ artiodáctilos (Familia Bovidae) (Muñoz et al., 2005; Esteves et al., 2013; Navarro et al., 2015).

En humanos (Villard et al., 2016), el diagnóstico serológico de toxoplasmosis se basa en la detección de niveles elevados de IgG, IgM e IgA anti-Toxoplasma. Entre estas, la IgM es la primera en incrementar (una semana posinfección), alcanzando su pico entre 1 y 3 meses, para disminuir durante los siguientes nueve meses hasta ser indetectable (aunque entre $9-27 \%$ de los pacientes pueden presentar IgM hasta por dos o más años).
La IgG es detectable a las dos semanas de la infección, con un pico a los tres meses, descendiendo lentamente desde el año hasta el fin de la vida del individuo (posiblemente debido a la persistencia de quistes). La IgA presenta una cinética similar a la IgM, pero con un pico más retrasado y persiste por tres a cuatro meses.

En primates no humanos infectados experimentalmente, se detectó $\operatorname{IgG}$ e $\operatorname{IgM}$ a partir del día nueve de la infección; sin embargo, IgM fue detectable solo hasta el día 13 (Bouer et al., 2010). En general, se asume que la IgM anti-Toxoplasma solo se detecta en la fase inicial de la infección y es útil para el diagnóstico de exposición reciente o enfermedad aguda, mientras que IgG sugiere enfermedad crónica (Yano et al., 1983; Sedlak y Bartova, 2006). Sin embargo, no existen estudios que detallen la cinética de la respuesta humoral anti-Toxoplasma en hurones. En el presente caso se detectaron títulos de Ig anti-Toxoplasma con y sin mercaptoetanol; resultado que se interpreta como detección de IgM y que sugiere el desarrollo de toxoplasmosis aguda.

La presentación clínica de toxoplasmosis en hurones es bastante variable, pudiéndose observar anemia, lesiones oculares (retinitis o iritis), hepatitis (con ictericia), ceguera, signos neurológicos, diarrea, signos respiratorios, letargia, fiebre y anorexia (Lewington, 2007). En el presente caso, solo se observó diarrea, letargia y anorexia, aunque también se observaron otros signos como bradicardia, hipotermia y deshidratación. Esta variabilidad e inespecificidad en la presentación clínica dificulta el diagnóstico clínico y demanda la consideración de otras enfermedades infecciosas de manifestación similar previamente observadas en hurones en Perú, como la coronavirosis sistémica (Lescano et al., 2015), entre los diagnósticos diferenciales.

Entre los hallazgos hematológicos destacan leucocitosis, neutrofilia y trombocitopenia. Sin embargo, la presencia de dos de estas alteraciones (leucocitosis y 
trombocitopenia) es cuestionable, ya que los rangos referenciales varían entre autores (Hein et al., 2012; Quesenberry y Orcutt, 2012). La única alteración hematológica aparentemente indiscutible es la neutrofilia, la cual podría asociarse al sobrecrecimiento bacteriano y enteritis (reflejada en el hallazgo de diarreas), signos que también han sido observados en casos de toxoplasmosis en hurones (Lewington, 2007).

La hipoglucemia observada podría explicarse por el periodo de inanición (posiblemente mayor de 6 horas) que afrontó el paciente antes de la toma de muestra (Mayer, 2008). Cabe señalar que la hipoglucemia asociada a insulinoma es un problema común en hurones mayores de 4 años (Mayer, 2008). Asimismo, otras causas de hipoglucemia en hurones son enfermedad hepática, neoplasias, hipoadrenocorticismo, sepsis y policitemia (Rosenthal, 2012).

El aparente incremento de la creatina quinasa observado en el presente caso puede ser debatible, ya que los rangos referenciales hallados en la literatura para este analito en Mustela putorius furo son considerablemente variables. Así, por ejemplo, Hein et al. (2012) presentan un rango entre 94 y $731 \mathrm{UI} / \mathrm{L}$ en muestras de suero, mientras que Heatley (2006) presenta un rango entre 98 y 564 UI/L (sin especificar si se empleó suero o plasma). Por otro lado, Quesenberry y Orcutt (2012) presentan un rango de 55 a $93 \mathrm{UI} / \mathrm{L}$ en muestras de plasma, mientras que Ohwada y Katahira (1993) presentan un rango de 164-274 UI/L en muestras de suero. Al respecto, Hein et al. (2012) destacan que estas diferencias podrían deberse a las metodologías de laboratorio empleadas y la población de animales muestreada (animales del laboratorio $v s$ animales mascota). El aparente incremento de la creatina quinasa observado sugeriría que el paciente presentaba necrosis a nivel de la musculatura esquelética (Garner et al., 2007).

Si bien no existe mucha información sobre las alteraciones bioquímico séricas aso- ciadas a toxoplasmosis en hurones, este mismo hallazgo en otros carnívoros domésticos es sugerente de toxoplasmosis o neosporosis muscular, aunque su presentación es poco frecuente (Lindsay et al., 1995; Barrs et al., 2006).

\section{Agradecimientos}

Los autores agradecen el apoyo brindado por la alumna Claudia Nolasco en el tratamiento del paciente, cuyo caso se presenta en este artículo.

\section{Literatura Citada}

1. Barrs VR, Martin P, Beatty JA. 2006. Antemortem diagnosis and treatment of toxoplasmosis in two cats on cyclosporine therapy. Aust Vet J 84: 30-35.

2. Bouer A, Werther K, Machado RZ, Nakaghi ACH, Epiphano S, CataoDias JL. 2010. Detection of antiToxoplasma gondii antibodies in experimentally and naturally infected non-human primates by Indirect Fluorescence Assay (IFA) and indirect ELISA. Rev Bras Parasitol Vet 19: 2631. doi: 10.4322/rbpv.01901005

3. Burns R, Williams ES, O'Toole D, Dubey JP. 2003. Toxoplasma gondii infections in captive black-footed ferrets (Mustela nigripes), 1992-1998: clinical signs, serology, pathology, and prevention. J Wildl Dis 39: 787-797. doi: 10.7589/0090-3558-39.4.787

4. Carpenter JW, Marion CJ. 2012. Exotic animal formulary. $4^{\text {th }}$ ed. San Luis, MO: Elsevier Saunders. 744 p.

5. Collins DM. 2015. Ursidae. In: Miller RE, Fowler ME (eds). Fowler's Zoo and Wild Animal Medicine. San Luis, MO: Elsevier Saunders. p 498-508.

6. Dubey JP, Odening K. 2001. Toxoplasmosis and related infections. In: Samuel WM, Pybus MJ, Kocan AA (eds). Parasitic diseases of wild mammals. Ames, IO: Iowa University Press. p 478-519. 
7. Esteves K, Chávez A, Casas E, Lí O. 2013. Determinación de anticuerpos contra Toxoplasma gondii en búfalos de agua (Bubalus bubalis) en el distrito de Jenaro Herrera, Loreto, Perú. Rev Inv Vet Perú 24: 390-395. doi: 10.15381/ rivep.v24i3.2589

8. García-Bocanegra I, Dubey JP, Martínez, Fargas A, Cabezón O, Zorrilla I, Arenas A, Almería S. 2010. Factors affecting serporevalence of Toxoplasma gondii in the endangered Iberian lynx (Lynx pardinus). Vet Parasitol 167: 36-42. doi: 10.1016/ j.vetpar.2009.09.044

9. Garner MM, Ramsell K, Schoemaker NJ, Sidor IF, Nordhausen RW, Bolin S, Evermann JF, Kiupel M. 2007. Myofascitis in the domestic ferret. Vet Pathol 44:25-38. doi: 10.1354/vp.44-1-25

10. Heatley JJ. 2006. Ferret cardiomyopathy. Compen Stand Care Emerg Crit Care Med 8(3): 7-11.

11. Hein J, Spreyer F, Sauter-Louis C, Hartmann K. 2012. Reference ranges for laboratory parameters in ferrets. Vet Rec 171: 218. doi: 10.1136/vr.100628

12. Lescano J, Quevedo M, Gonzales-Viera O, Luna L, Kell MK, Gregori F. 2015. First case of systemic coronavirus infection in a domestic ferret (Mustela putorius furo) in Peru. Transbound Emerg Dis 62: 581-585. doi: 10.1111/ tbed. 12407

13. Lewington JH. 2007. Parasitic diseases of ferrets. In: Lewington JH (ed). Ferret husbandry, medicine and surgery. $2^{\text {nd }} \mathrm{ed}$. China: Saunders. p 224-257.

14. Lindsay DS, Blagburn BL, Braund KG. 1995. A review of Toxoplasma gondii and muscular toxoplasmosis. Basic Appl Myol 5: 255-260.

15. Mayer J. 2008. Interpreting the chemistry profile in ferrets. Proc North American Veterinary Conference. Orlando, FL, USA.

16. McDonald RA, Larivière S. 2001. Disease and pathogens of Mustela spp, with special reference to the biological control of introduced stoat Mustela ermine populations in New Zealand. J Roy Soc New Zeal 31: 721-744.

17. Mentré $V$. 2012. Un caso di toxoplasmosis nel furetto. It J Companion Anim Pract 1(1): 26-27.

18. Miller MA. 2008. Tissue cyst-forming coccidia of marine mammals. In: Fowler ME, Miller RE (eds). Zoo and wild animal medicine: current therapy 6 . San Luis, MO: Saunders Elsevier. p 319-340.

19. Morrisey JK. 1996. Parasites of ferrets, rabbits, and rodents. Semin Avian Exotic Pet Med 5: 106-114. doi: 10.1016/S1055937X(96)80023-5

20. Muñoz E, Chávez A, Casas E, Suárez F, Gavidia C, Muñoz K, Gutiérrez F. 2005. Frecuencia de anticuerpos contra Toxoplasma gondii en monos Cebus apella criados en cautiverio. Rev Inv Vet Perú 16: 163-168. doi: 10.15381/ rivep.v16i2.1565

21. Navarro D, Chávez A, Pinedo R, Muñoz K. 2015. Factores de riesgo associados a la seroprevalencia de Toxoplasma gondii em mamíferos del Orden Carnivora y Primates mantenidos en cautiverio. Rev Inv Vet Perú 26: 497508. doi: 10.15381/rivep.v26i3.11175

22. O'Crowley K, Wilson JG 1991. Feral mink (Mustela vison) and their potential as disease vectors in Ireland: an investigation in Co. Wicklow. Ir Vet J 44: 71-74.

23. Ohwada K, Katahira K. 1993. Reference values for organ weight, hematology and serum chemistry in the female ferret (Mustela putorius furo). Jikken Dobutsu 42: 135-142.

24. Onuma SSM, Melo ALT, Kantek DLZ, Crawshaw-Junior PG, Morato RG, May-Júnior JA, Pacheco TA, Aguiar DM. 2014. Exposure of free-living jaguars to Toxoplasma gondii, Neospora caninum and Sarcocystis neuro$n a$ in the Brazilian Pantanal. Rev Bras Parasitol Vet 23: 547-553. doi: 10.1590/ S1984-29612014077 
25. Quesenberry KE, Orcutt C. 2012. Basic approach to veterinary care. In: Quesenberry KE, Carpenter JW (eds). Ferrets, rabbits, and rodents: clinical medicine and surgery. $3^{\text {rd }}$ ed. San Luis, MO: Elsevier Saunders. p 13-26.

26. Rosenthal K. 2012. Endocrine diseases. In: Quesenberry KE, Carpenter JW (eds). Ferrets, rabbits, and rodents: clinical medicine and surgery. $3^{\text {rd }}$ ed. San Luis, MO: Elsevier Saunders. p 86-102.

27. Sedlak K, Bartova E. 2006. The prevalence of Toxoplasma gondii IgM and $\mathrm{IgG}$ antibodies in dogs and cats from the Czech Republic. Vet Med 51: 555558.

28. Smielewska-Los E, Matusiewicz K, Wincewicz E, Klimentowski S. 2000. Diagnosis of Toxoplasma gondii infections in newborns farm foxes by polymerase chain reaction (PCR). Scientifur 24: 233-238.

29. Sobrino R, Cabezón O, Millán J, Pabón M, Arnal MC, Luco DF, Gortázar C, et al. 2007. Seroprevalence of Toxoplasma gondii antibodies in wild carnivores from Spain. Vet Parasitol 148: 187-192. doi: 10.1016/j.vetpar.2007.06.038

30. Tenter AM, Heckeroth AR, Weiss LM. 2000. Toxoplasma gondii: from animals to humans. Int J Parasitol 30: 1217-1258.
31. Thornton RN, Cook TG. 1986. A congenital Toxoplasma-like disease in ferrets (Mustela putorius furo). $\mathrm{N} \mathrm{Z}$ Vet J 34(3): 31-33. doi: 10.1080/ 00480169.1986 .35267

32. Tizard IR, Billet JB, Ramsden RO. 1976. The prevalence of antibodies against Toxoplasma gondii in some Ontario mammals. J Wildl Dis 12: 322325.

33. Tocidlowski ME, Lappin MR, Sumner PW, Stoskopf MK. 1997. Serologic survey for toxoplasmosis in river otters. J Wildl Dis 33: 649-652. doi: 10.7589/ 0090-3558-33.3.649

34. Villard O, Cimon B, L'Ollivier C, Fricker-Hidalgo $H$, Godineau $N$, Houze S, Paris L, et al. 2016. Serological diagnosis of Toxoplasma gondii infection: recommendations from the French National Reference Center for Toxoplasmosis. Diagn Microbiol Infect Dis 84: 22-33. doi: 10.1016/ j.diagmicrobio.2015.09.009

35. Yano A, Yui K, Yamamoto M, Aosai F, Furuta S, Kojima S. 1983. Immune response to Toxoplasma gondii. I. Toxoplasma-specific proliferation response of peripheral blood lymphocytes from patients with toxoplasmosis. Microbiol Immunol 27: 455-463. doi: 10.1111/j.1348-0421.1983.tb00604.x 